M. Steger, G. Abagnale, E. Bremus-Köbberling, W. Wagner, and A. Gillner

\title{
2 Nanoscale biofunctionalization of polymer surfaces by laser treatment for controlled cellular differentiation
}

\begin{abstract}
In this chapter we will discuss how nanostructures can interact with stem cells. After an introduction regarding the basic concepts of stem cells, their properties, and their uses in the medical field, we will then examine how stem cells can recognize physical forces and how this can be reproduced in vitro using materials produced in micro- and nanofabrication processes. Afterwards we will describe the principles and applications of a novel direct laser ablation technique, multibeam interference, and how patterns produced with such a method can be employed in stem cell research.
\end{abstract}

\subsection{Nanostructuring and mesenchymal stem cells}

\subsubsection{Introduction to stem cells}

The heterogeneity of human cells is extremely broad; nonetheless, all cells can be roughly divided into two categories: differentiated cells which perform specific, defined functions, and stem cells which represent the undifferentiated, unspecialized progenitors whence all somatic cells originate. During intrauterine life stem cells are responsible for the development of the human being from zygote to completely formed fetus, whereas in the adult individual they control wound repair and the general homeostasis of tissues, e.g. the regrowth of hair and nails [1-3].

\subsubsection{Definition of stemness}

Stem cells are generally characterized by two key features: their differentiation potential and their self-renewal ability $[4,5]$. The broadness of somatic cell types which can originate from stem cells is indicative of their potency. The more a stem cell is committed toward a defined fate, the less its potency (Fig. 2.1 (a)). Additionally, stem cells must possess self-renewal capacity to keep their number constant. When stem cells undergo cell division, the process can be either symmetric or asymmetric (Fig. 2.1 (b)). The balance between symmetric and asymmetric division affects self-renewal and differentiation of stem cells [6]. This complicated decision is regulated by an intricate network of biochemical and physical stimuli present in the milieu in which such cells are embedded: the stem cell niche [7]. 
(a)

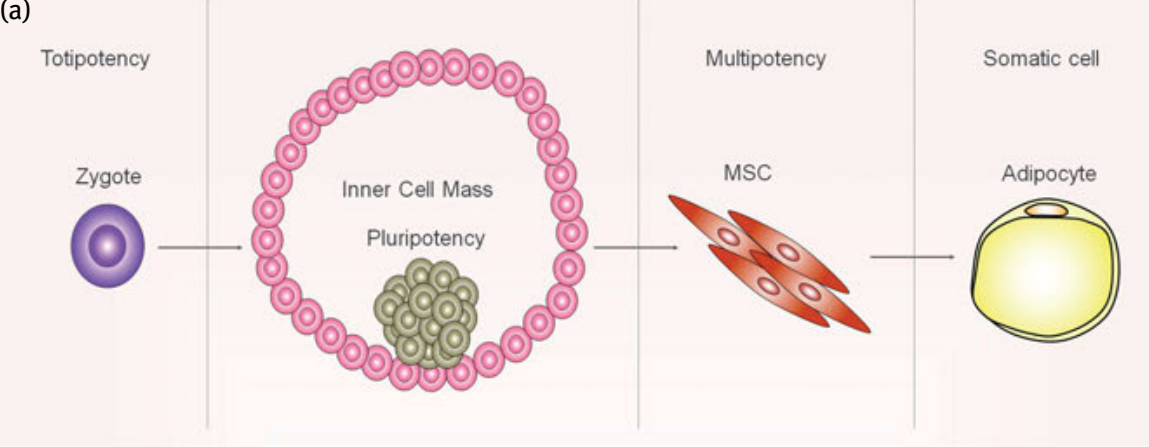

(b)

Symmetric divisions
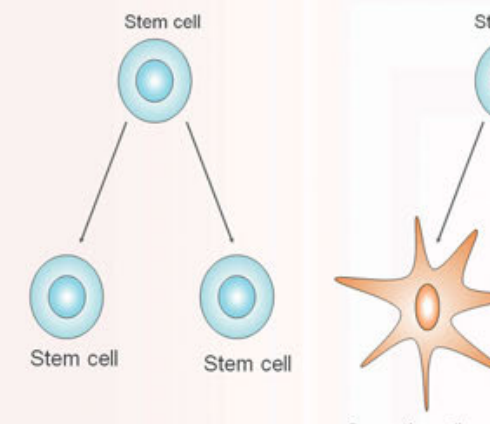

Somatic cell
Stem cell

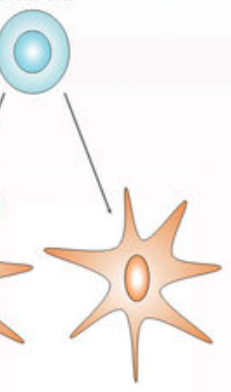

Somatic cell
Asymmetric division

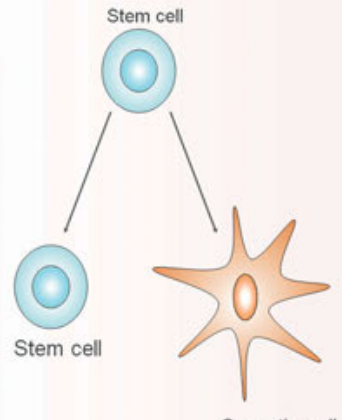

Somatic cell

(c)

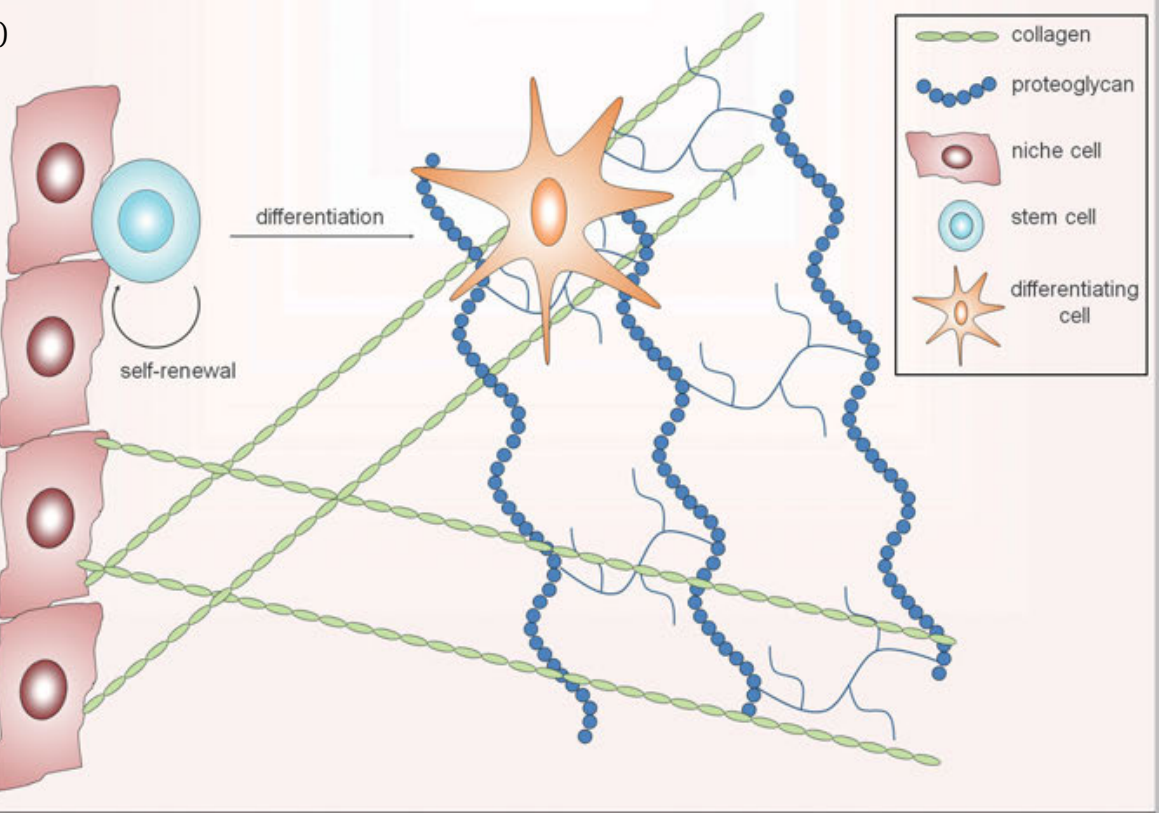




\subsubsection{The stem cell niche}

The stem cell niche is the microenvironment which supports stem cell survival and self-renewal [8]. This niche can be composed of both cellular and non-cellular elements as extracellular matrix proteins or the basement membrane. Cell-cell and cellmatrix interactions in combination with soluble cytokines and growth factors contribute to the interplay between stem cells and their niche (Fig. 2.1 (c)). This tight signaling and the physical support provided by the niche modulate processes such as tissue regeneration and cellular turnover [9].

\subsubsection{Mesenchymal stem cells}

\subsubsection{Introduction to MSCs}

Mesenchymal stem cells (MSCs) can be found in several anatomical locations, though they are more abundant in bone marrow and adipose tissue [10, 11]. MSC preparations differ consistently depending on the site of origin, age, and sex of the donor $[12,13]$. Nonetheless, MSCs are characterized by some common features: (1) they display good adherence to tissue culture plastic, exhibit a fibroblast-like morphology and can form colonies in vitro. (2) MSCs are usually positive for the surface antigens STRO-1 and CD73, while they do not normally express the lymphoid marker CD45 and the hematopoietic marker CD34. (3) MSCs are believed to be multipotent since they can differentiate into cell types deriving from a single embryonic layer, the mesoderm [14-16].

\subsubsection{Functions of MSCs}

One of the crucial functions of MSCs is to maintain homeostasis in tissues of mesodermal derivation. In fact, it has been demonstrated that MSCs can give rise in vitro to osteocytes, adipocytes, chondrocytes, fibroblasts, tenocytes, endothelial cells, smooth muscle cells, myoblasts, and cardiomyocytes [17, 18]. Another important function of MSCs is their immunomodulatory ability. Many studies have shown that MSCs can regulate the immune effectors through secretion of cytokines and direct interaction with immunocompetent cells. In detail, MSCs are able to dampen both adaptive and innate responses, and hence shift the balance of immune system activation toward an anti-inflammatory and immunotolerant state [19].

4 Fig. 2.1: Schematic presentation of stem cell function. (a) The differentiation potential of a stem cell determines its potency. (b) The balance between symmetric and asymmetric cell divisions regulates stem cell self-renewal. (c) Schematic representation of a putative niche for stem cells comprising cellular (stromal cells) and noncellular elements (extracellular matrix proteins). 


\subsubsection{Clinical relevance of MSCs}

MSCs can be used in clinical practice, e.g. their immunomodulatory properties could be exploited to improve transplant engraftment or treat chronic inflammatory diseases. Moreover, the ability of MSCs to differentiate into cells of mesodermal lineage makes them the perfect candidate for tissue engineering applications. MSCs have been used to repair cartilage or bone defects, either by applying the cells to the site of injury directly or after culture on biomimetic scaffolds to facilitate regeneration and integration into the surrounding tissue [20]. Most efforts to improve MSC-based regenerative therapies have focused on coordinating chemical and biological signals to achieve controlled cellular differentiation. An increasing number of studies have recently indicated that the effects of physical forces on cell dynamics might be the missing link in achieving this aim [21].

\subsubsection{Mechanobiology of MSCs}

\subsubsection{Mechanical stimuli in the stem cell niche}

Mechanobiology is the area of cellular biology which focuses on how cells recognize mechanical forces and then transduce them into a biological response [22]. Inside their niche MSCs are subjected to several types of physical stimuli: (1) shear stress generated by the blood flow, which is particularly relevant for perivascular MSCs [23, 24]; (2) mechanical strain associated with the traction of muscles and bone [25, 26]; (3) substrate stiffness, which has a deep impact on MSC behavior [27, 28]. Interestingly substrate stiffness also has an effect on MSC motility, since it has been shown that cells migrate from softer to stiffer areas in a process called durotaxis [29]. (4) Surface topography. In fact, the stem cell niche is a 3D microenvironment which includes structures of different sizes, shapes, and spatial disposition [30]. Following the physical interaction with their substrate, cells are able to first "sense" and distinguish diverse topographic cues and then integrate them into a specific biological response [31].

\subsubsection{The mechanotransducer machinery of the cell}

Integrins are a family of receptor proteins found on the cellular membrane and responsible for the mechanical anchorage of a cell to its substrate [32]. ECM proteins are the main ligand of integrin receptors.The binding of an integrin to its ligand elicits a complex response which leads to the formation of focal adhesions [33-36]. Focal adhesions recognize mechanical forces generated either by an external source or by the cell itself, and then translate them into a biological response (Fig. 2.2). Following the binding of their extracellular portions to the ECM, the cytoplasmic tails of the integrins recruit the protein talin, which functions as bridge between the site of adhesion and the cytoskeleton since it binds actin fibers both directly and through the protein vinculin. Anchorage of the actin filaments to the site of adhesion generates 
internal mechanical forces and triggers integrin clustering, a fundamental event in the genesis of focal adhesions. Clustered integrins recruit more actin filaments to the site of adhesion, thus leading to the formation of contractile structures composed of actin microfilaments connected by myosin II molecules called stress fibers. This initial integrin/cytoskeleton complex evolves into a mature focal adhesion after the recruitment of various additional proteins including paxillin, zyxin, and the tyrosine kinase FAK. The signaling cascades downstream of focal adhesions include the activation

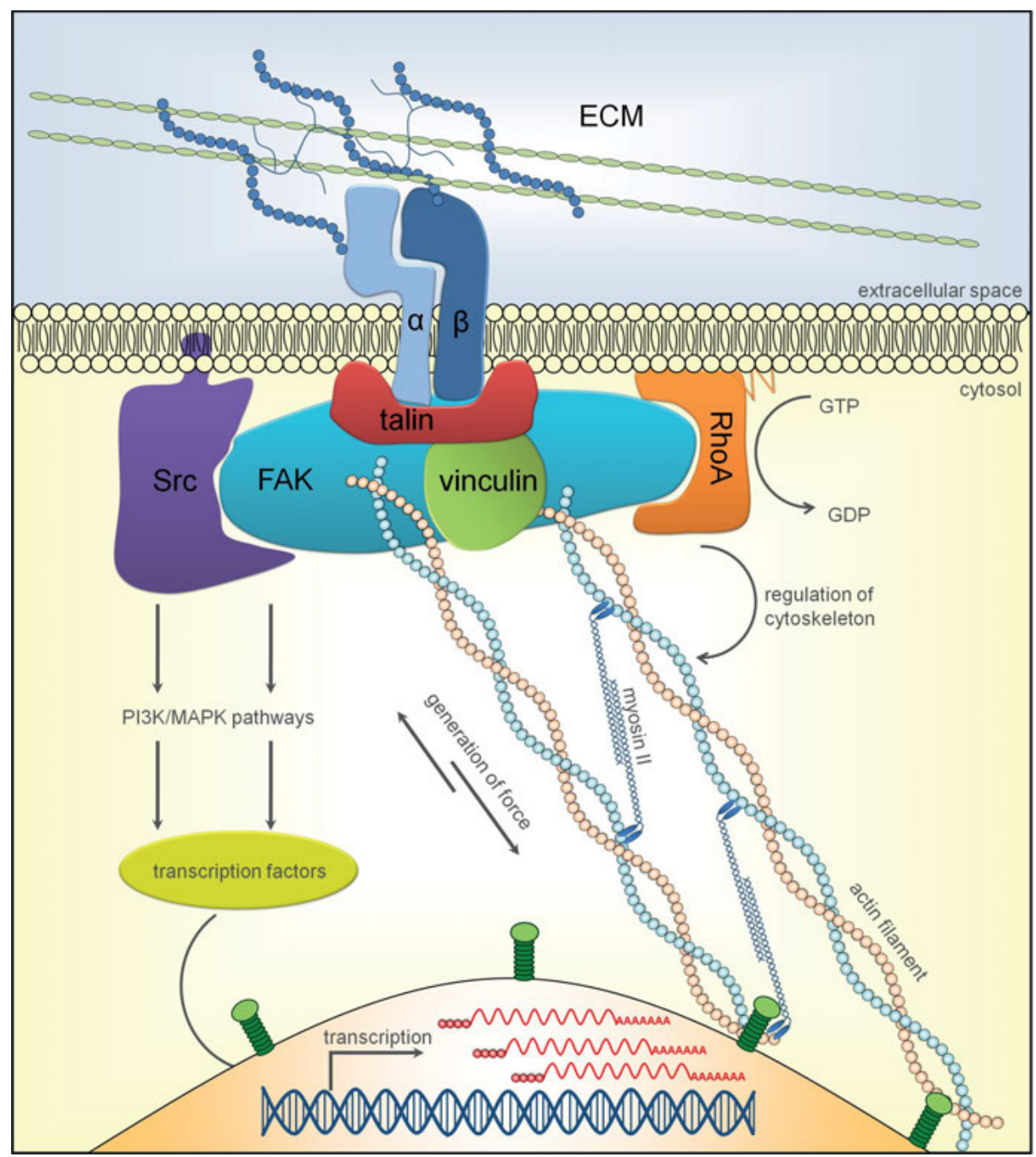

Fig. 2.2: Integrin-mediated recognition of extracellular matrix components leads to the formation of focal adhesion complexes able to connect the cytoskeleton with the adhesion site and moreover triggers downstream signaling pathways. 
of the MAPK/ERK pathway, resulting in transcriptional regulation of cell cycle and differentiation, and the phosphorylation of the small GTPases of the Rho family, which regulate cytoskeletal dynamics and cell motility [37].

\subsubsection{Interaction of MSCs with micro and nanostructures}

Biomaterials are the ideal tool for studying the effects of substrate stiffness and surface topography on MSCs [38, 39]. Defined micro- and nanostructures have been shown to have a prominent role in keeping MSCs in a quiescent multipotent state or in directing their differentiation towards specific fates [40-43]. Fibrils, pillars, pits, grooves, and ridges are the features which better resemble the topographical landscape found in the stem cell niche; nonetheless the variety of cues which can be studied is much broader. In fact, it should be considered that scale size and the symmetric disposition of the structures contribute to increasing the combination of conceivable theoretical patterns [44, 45]. In this scenario the only limit to the number of cues which we can analyze is presented by our technological progress. In this regard, the ideal patterning process should allow generation of high resolution structures in a fast, cost-efficient, and reproducible fashion.

\subsection{Nanostructuring of materials}

\subsubsection{Direct nanostructuring of surfaces with multibeam-interference}

The most commonly used method for nanostructuring of surfaces is lithography. For more than three decades lithography has been the key enabling technology to decrease the structure size in semiconductors manufacturing to fulfill Moore's law [46] and sub $100 \mathrm{~nm}$ structuring by lithographic had its industrial breakthrough around 2005. The drawback of high resolution lithography is its high price, due to the many complex process steps and high precision photo masks. Therefore a crucial aim in ongoing research is not only the further reduction of structure size, but also cost reduction. Besides the reduction of the necessary steps involving lithography, in nanoimprinting, for example, multibeam interference lithography (MBI-L) is a promising approach as a flexible substitute for expansive photo masks. The periodic interferences pattern created by two or more coherent laser beams is used to expose the photo resist in MBI-L and parallel to further research the first commercial applications are beginning to emerge. MBI-L also makes fabrication of 3D structures possible. Multibeam interference (MBI) is not, however, limited to the low energy illumination necessary for the chemical modification of photoresists, it can also be used for direct photoablation of many materials [47]. 
The direct nanostructuring of surfaces with multibeam interference therefore has several advantages. Structuring is carried out in a single step process and a whole surface area is structured simultaneously. The size of the area depends mainly on beam diameter and ablation threshold of the material. Periodicity and geometry of the structures generated can easily be adapted by changing alignment or polarization of beams [48]. Depending on the wavelength of the laser source, the size of the generated structures can range from the sub $100 \mathrm{~nm}$ up to several $\mu \mathrm{m}$. Many materials can also be processed with MBI, especially with use of ultra-short pulse (USP) laser sources. Its ability to structure surfaces cost-efficiently in a single step and its easy adaption of the pattern generated make MBI a very promising tool for cheap nanostructuring with periodic patterns, not only for research purposes, but also for industrial applications. Additionally, its inherent ability to structure nonplane surfaces is an interesting feature compared to standard lithography [49].

\subsubsection{Theoretical background}

The capacity for structuring by multibeam interference is based on the intensity modulation caused by the interference of two or more intercepting coherent laser beams. This intensity modulation is present in the whole volume where the beams intercept and the total time independent intensity modulation $I(\boldsymbol{r})$ inside this volume of the number of $j$ plane waves can be described as follows [50]:

$$
I(\boldsymbol{r})=\sum_{j} I_{j}(\boldsymbol{r})+2 \sum_{i<j} \sqrt{I_{i}(\boldsymbol{r}) I_{j}(\boldsymbol{r})} \cos \left(\theta_{i j}\right) \cos \left(\left(\boldsymbol{K}_{i}-\boldsymbol{K}_{j}\right) \boldsymbol{r}+\phi_{i}-\phi_{j}\right),
$$

where $I_{j}$ is the intensity of beam $j, \theta_{i j}$ is the angle between the unit vectors of beam $i$ and $j$ in its polarization direction, $\boldsymbol{K}_{j}$ is the wave vector of beam $j$ and the phase of beam $i$. The first sum of equation (2.1) describes the total intensity of all beams without interference, while the second sum defines the modulation around due to the interference. For a two-beam interference setup the expression can be written as:

$$
I_{2 B}(\boldsymbol{r})=I_{1}+I_{2}+2 \sqrt{I_{1} I_{2}} \cos \left(\theta_{12}\right) \cos \left(\left(\boldsymbol{K}_{1}-\boldsymbol{K}_{2}\right) \boldsymbol{r}+\phi_{i}-\phi_{j}\right),
$$

where $\omega$ is the beam radius. Further simplified for identical amplitude, polarization, and initial phase of both beams with $\lambda$ as wavelength of the laser and $\alpha$ the half intersection angle of both beams, equation (2.2) can be simplified to:

$$
I_{2 B}(\boldsymbol{x})=2 I_{1}\left(1+\cos \left(x \frac{2 \pi}{\lambda} \sin \alpha\right)\right) .
$$

The intensity pattern generated is in the shape of cosine in the direction of the $x$-axis, while the pattern is constant in $y-z$ plane. As visible in equation (2.3), the periodicity of this modulation can be controlled by the wavelength $\lambda$ and the intersection angle $\alpha$. This correlation is shown in Fig. 2.3 (a) and 2.3 (b). The ideal modulated intensity reaches twice the intensity $I_{0}$ in its maxima $I_{\max }$, and zero intensity in its minima $I_{\min }$. 
The depth of modulation compared to the ideal modulation depends on the coherence of the beams and their ability to interfere, and can be described by the interference contrast as:

$$
\text { Contrast }=\frac{I_{\max }-I_{\min }}{I_{\max }+I_{\min }}
$$

It ranges from 1 for an ideal modulation and 0 for an unmodulated signal (compare Fig. 2.3 (c) and 2.3 (d)). A measuring method for the interference contrast was developed and tested as part of this project [51]. High contrast is important for the optimization of direct surface structuring by MBI, because its value is directly linked to the quality of the ablation process. Reduced contrast means a decrease of $I_{\max }$ and an increase of $I_{\min }$, and therefore leads to a shift in intensity distribution from the ablation areas to the areas where no material alteration is desired. As a result of this shift, a reduced ablation depth is reached for a fixed total intensity and increased heating, ablation, or even destruction of the whole pattern occurs by the higher intensity of $I_{\min }$. For these reasons, the maximal ablation depth, structure size, and periodicity of the generated patterns is closely coupled to the interference contrast.
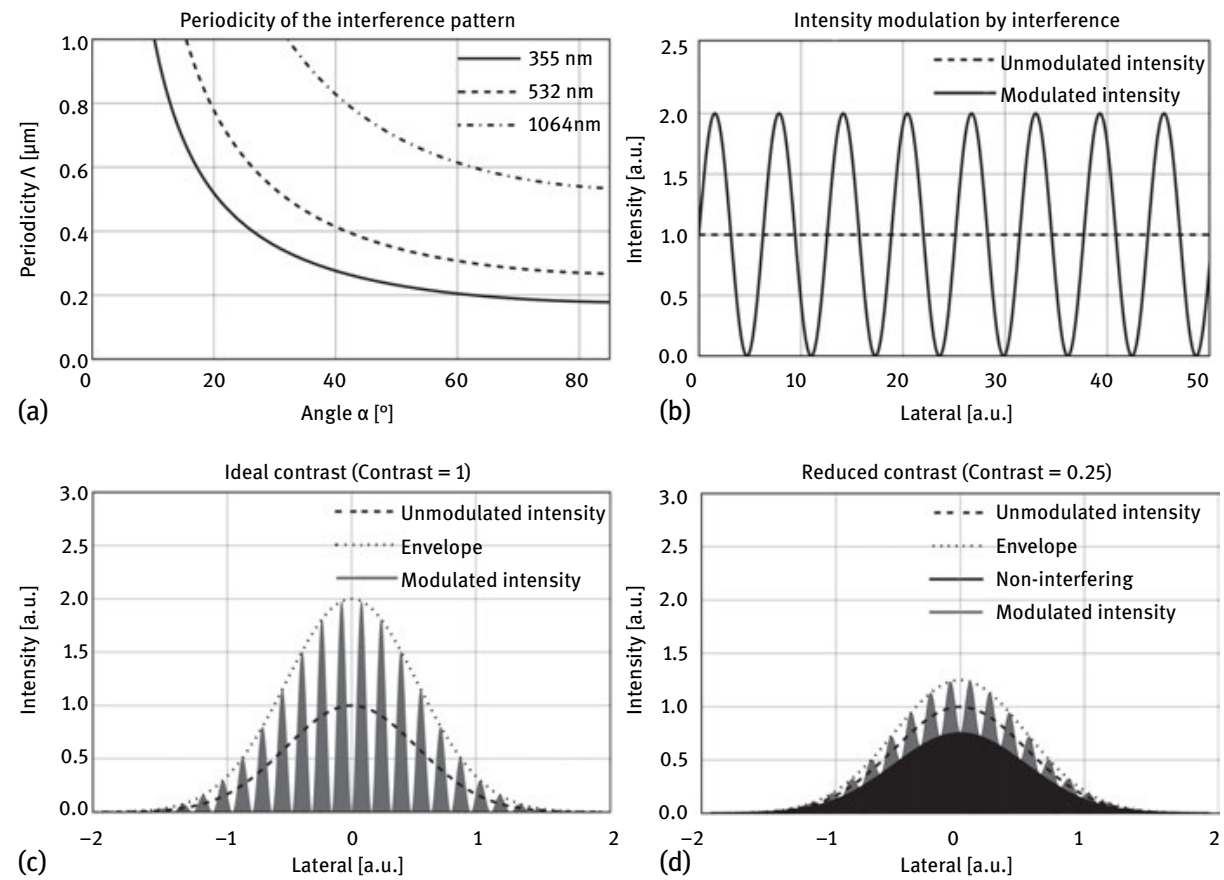

Fig. 2.3: Characteristics of 2-beam interference: (a) periodicity of a 2-beam interference pattern depending on the intercepting angle for 3 different wavelengths; (b) intensity modulation created by the interference of two beams compared to unmodulated signal; (c) intensity modulation of a Gaussian beam shape for an ideal contrast; (d) intensity modulation of a Gaussian beam shape for a reduced contrast. 


\subsubsection{Experimental setup \& methods}

The laser source is a diode pumped Nd:YAG laser (Q301-HD, JDSU) which operates at a wavelength of $355 \mathrm{~nm}$ with a pulse duration of $38 \mathrm{~ns}$ and a coherence length of $5.4 \mathrm{~mm}$. The polarization of the laser beam is turned to a perpendicular polarization by a half-wave plate in relation to the plane of the optical table. Afterwards the beam is refined through a spatial room filter and split into two parts. Two different setups were used as beam splitters: first a reflective $50: 50$ beam splitter and second a transmissive diffractive grating. The diffractive grating has an efficiency of $78 \%$ in the $1 /-1$ order used for the experiments. All other orders were blocked. Afterwards the partial beams are directed by mirrors to superpose at the sample surface. The intersection angle can be adjusted to control the periodicity of the applied pattern. The sample material is polyimide foil (Upilex), $50 \mu \mathrm{m}$ thick. The output of the laser is measured by an energy detector (QE12LP-S-MP, Gentec) and a beam profiler (Spiricon, Ophir) at the intersection plane of the beams. A typical beam diameter is $\sim 900 \mu \mathrm{m}$ with maximum pulse energy of $1.2 \mathrm{~mJ}$. The samples are structured by a single pulse. The diameter of the area ablated by one pulse is about $600 \mu \mathrm{m}$. Larger areas of structured surfaces for cell seeding are stitched together in a close package. The geometry of the structures generated is measured by an atomic force microscope (Rados N8, Bruker) and a scanning electron microscope (LEO, Zeiss).

\subsubsection{Structuring results of multibeam interference}

The structures generated by a two-beam interference for a single step process is a 1D line with geometry as shown in Fig. 2.4. The periodicity of the line pattern can easily be controlled by adjusting the intersection angle between $210 \mathrm{~nm}$ and several $\mu \mathrm{m}$. The main structure size respective of the width of line generated depends on the fluence of the laser beam, the ablation threshold of the material, and the heat conductivity of the material. The width of the line can be varied in the wide regime for fixed periodicity of the interference pattern; see Fig. 2.4.

The interaction of the cells with the topography seems to be primarily at the elevated part of the structure and therefore not at the ablated surface area. As visible in the AFM scan in Figs. 2.4 and 2.5, an additional bulging at the edges of the generated grooves occurs. These bulges have a significant height and their size is only a fraction of the periodicity of the pattern. Due to their position they act as an additional divider between the elevated part of the surface and the ablated grooves.

A close package of the functional structure elements is necessary for to optimize the effect of cell guiding by surface topography. Therefore, even if sub $100 \mathrm{~nm}$ structures are reachable with a periodicity of $1000 \mathrm{~nm}$, the low density of the structures is problematic and reduces the relative influence on the growth and differentiation process of the stem cells. To increase the density it is necessary to decrease the periodicity and therefore raise the package density of the functional structure elements. In contrast, we observed that with a reduction of periodicity two kinds of problems occur, 

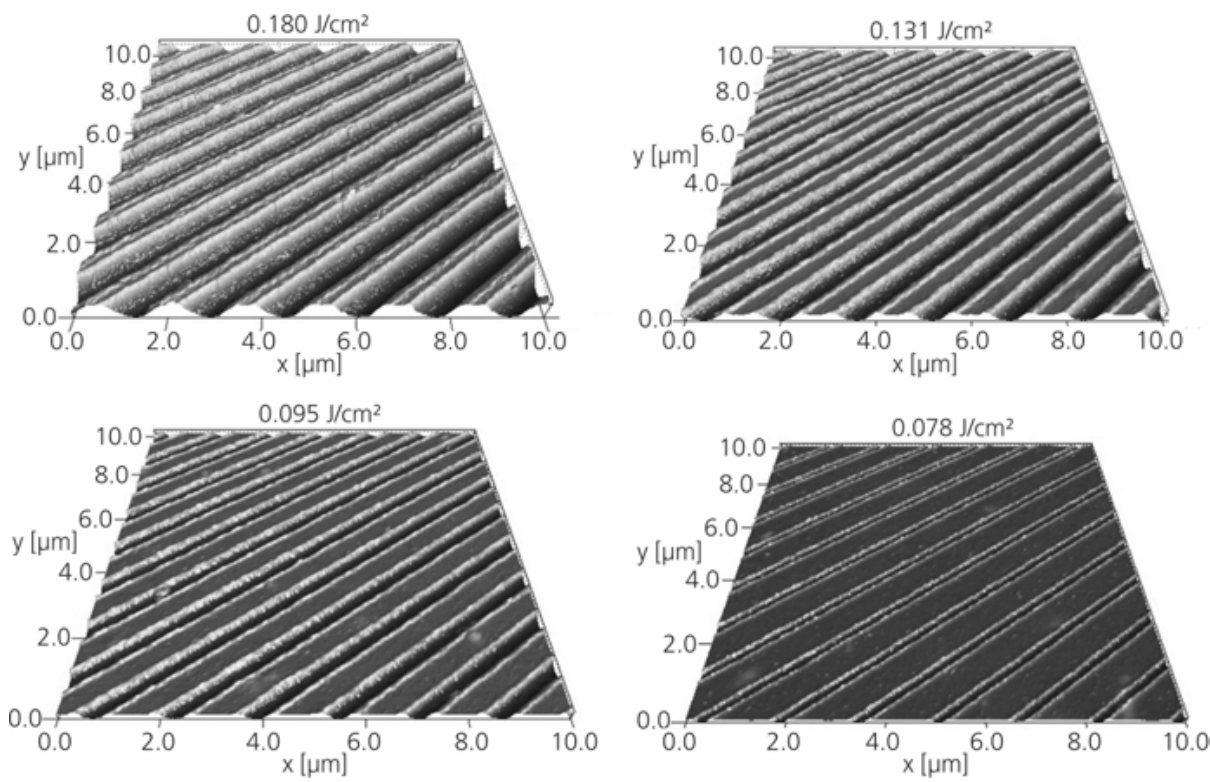

Fig. 2.4: AFM measurements of structured PI samples with different fluence. While the periodicity is constant with $1 \mu \mathrm{m}$, the geometry of generated grooves varies depending on the fluence.

or at least their influence on the structuring result increases: stability of structures and the homogeneity of the pattern. The stability of structures limits their maximal depth, which also has an important influence on cell growth and the homogeneity of pattern influences the structure deviations inside the pattern. Apart from this consideration we reached a minimal periodicity of $220 \mathrm{~nm}$ for the line pattern which leads to ridge width of less than $100 \mathrm{~nm}$ and bulges around $50 \mathrm{~nm}$ wide. An example for the structuring results is shown in Fig. 2.5.

\subsection{Impact of laser-ablated nanogrooves on cell biology}

\subsubsection{Cell guidance along nanogrooves}

The impact of laser-generated nanogrooves in the range of several hundred nanometers on neuronal cells has been shown in previous studies [52, 53]. Cellular extensions of neuronal B35 cells preferentially orient themselves parallel (i.e. within angles of 0 to $30^{\circ}$ ) to nanogrooves prepared via interference patterning. Our findings indicate that feature width is less crucial for alignment. Cells align along grooves which are $250 \mathrm{~nm}$ wide as well as along grooves $500 \mathrm{~nm}$ wide, but structure depth plays a crucial role. A depth to width (aspect) ratio greater than 0.6 induces a significantly higher degree of alignment compared to the shallower grooves. 
(b) Height profile
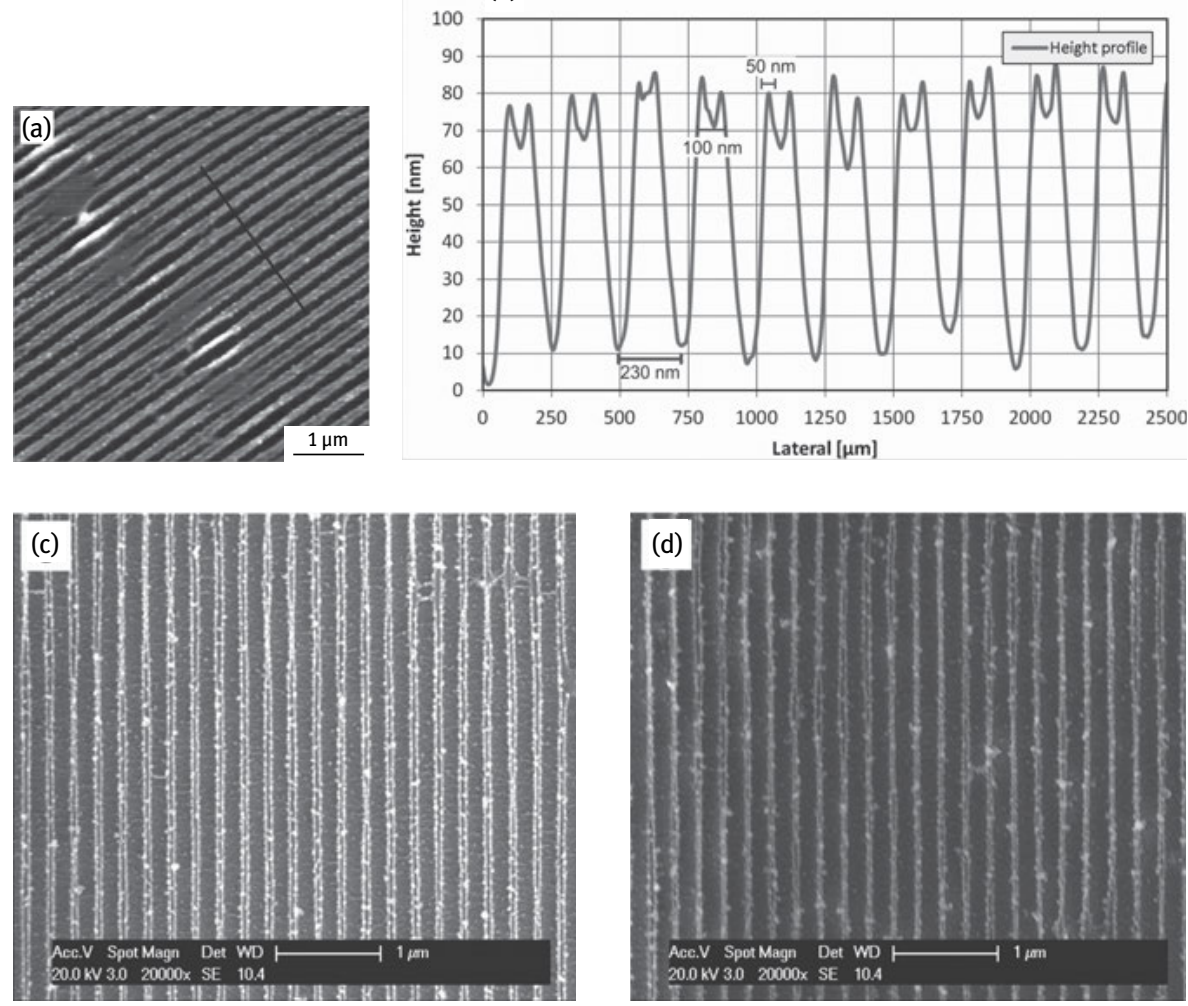

Fig. 2.5: (a) AFM measurements of structured PI samples with a periodicity of $230 \mathrm{~nm}$; (b) corresponding height profile extracted from subfigure (a); (c), (d) REM measurements of structured PI samples with a periodicity of $230 \mathrm{~nm}$.

In recent studies we examined the influence of three different nano patterns on orientation and length of cellular extensions. The nano patterns investigated were grooves, dots, and a mixture of both features called "dotted grooves" (compare Fig. 2.6) generated via interference patterning of polyimide (PI) and subsequent casting with polydimethylsiloxane (PDMS). PDMS casting was performed once to obtain a negative structure of the PI master and a second cast yielded the positive structure in PDMS. A loss in feature depth occurs due to the casting process. Casted groove structures are approx. 60 to $50 \%$ of original feature depth of PI master (e.g. $382 \mathrm{~nm}$ in $\mathrm{PI}$ and 244/194 nm in PDS cast one/two; cf. Fig. 2.7: AFM/SEM pictures). The samples were incubated with B 35 neuronal cells (3000 cells per $\mathrm{cm}^{2}$ ) and cell extensions were measured after two days of culture, followed by fixation with $4 \%$ formalin and actin/DAPI staining. No alignment of cellular extensions could be detected on the dotted samples (b) and (c) (Fig. 2.6). Due to the lower degree of regularity on these samples, dendrites orient right or left around the dots leading to a random distribution 

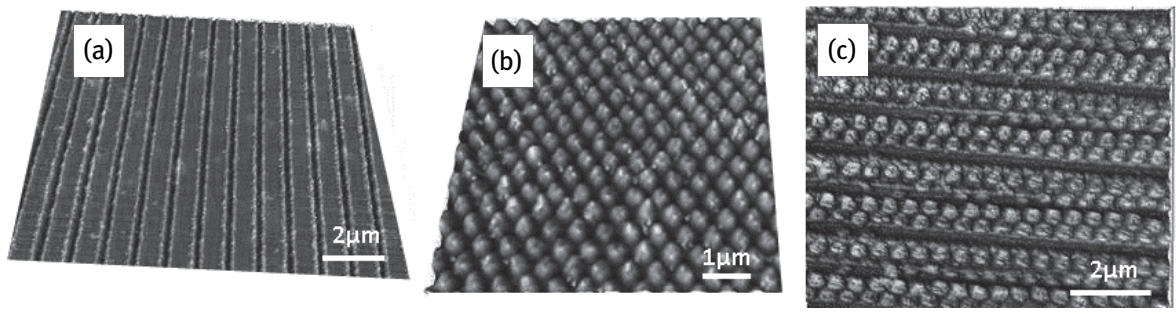

Fig. 2.6: AFM measurements of different nanopatterns: (a) grooves with $900 \mathrm{~nm}$ plateau and $200 \mathrm{~nm}$ groove width; (b) dots with $200 \mathrm{~nm}$ diameter and $200 \mathrm{~nm}$ pitch; (c) dotted grooves with two lines of dots per $900 \mathrm{~nm}$ plateau and $200 \mathrm{~nm}$ groove.

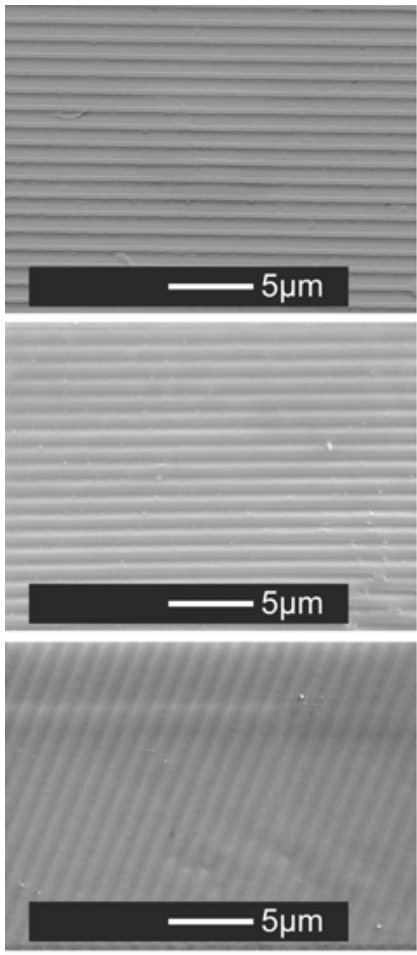

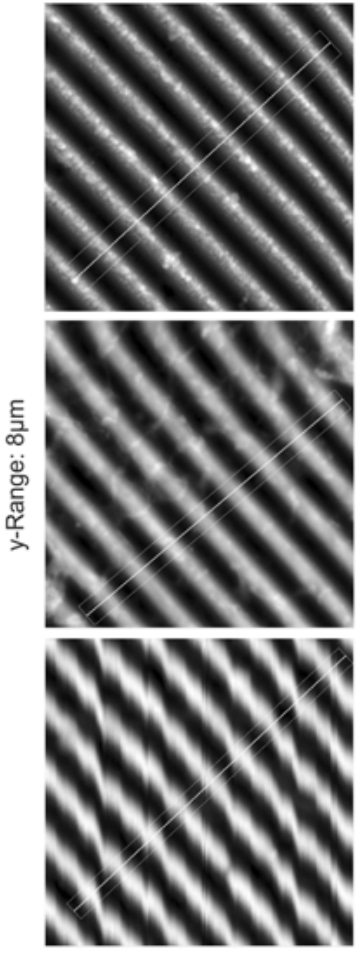

$\mathrm{x}$-Range: $8 \mu \mathrm{m}$
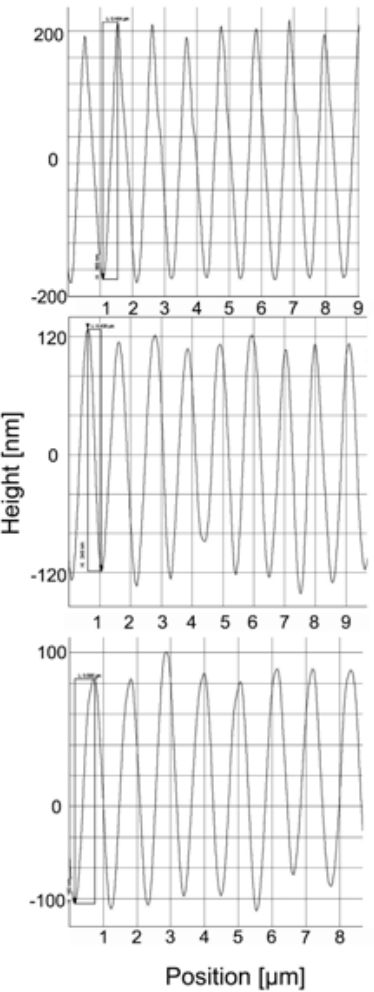

Fig. 2.7: AFM/SEM of nanogrooves. PI directly structured, PDMS-nL: cast one, negative structure of $\mathrm{PI}$ and PDMS-pL: cast two, shallow positive structure.

of cell extensions. The following observations were made regarding cell numbers and lengths of dendrites (Fig. 2.8):

- less cells on unstructured polymer surfaces than on glass positive reference

- longest cell extensions on glass positive reference (maximum length on glass 40-60 $\mu \mathrm{m}$, maximum length on polymers $20-40 \mu \mathrm{m})$ 


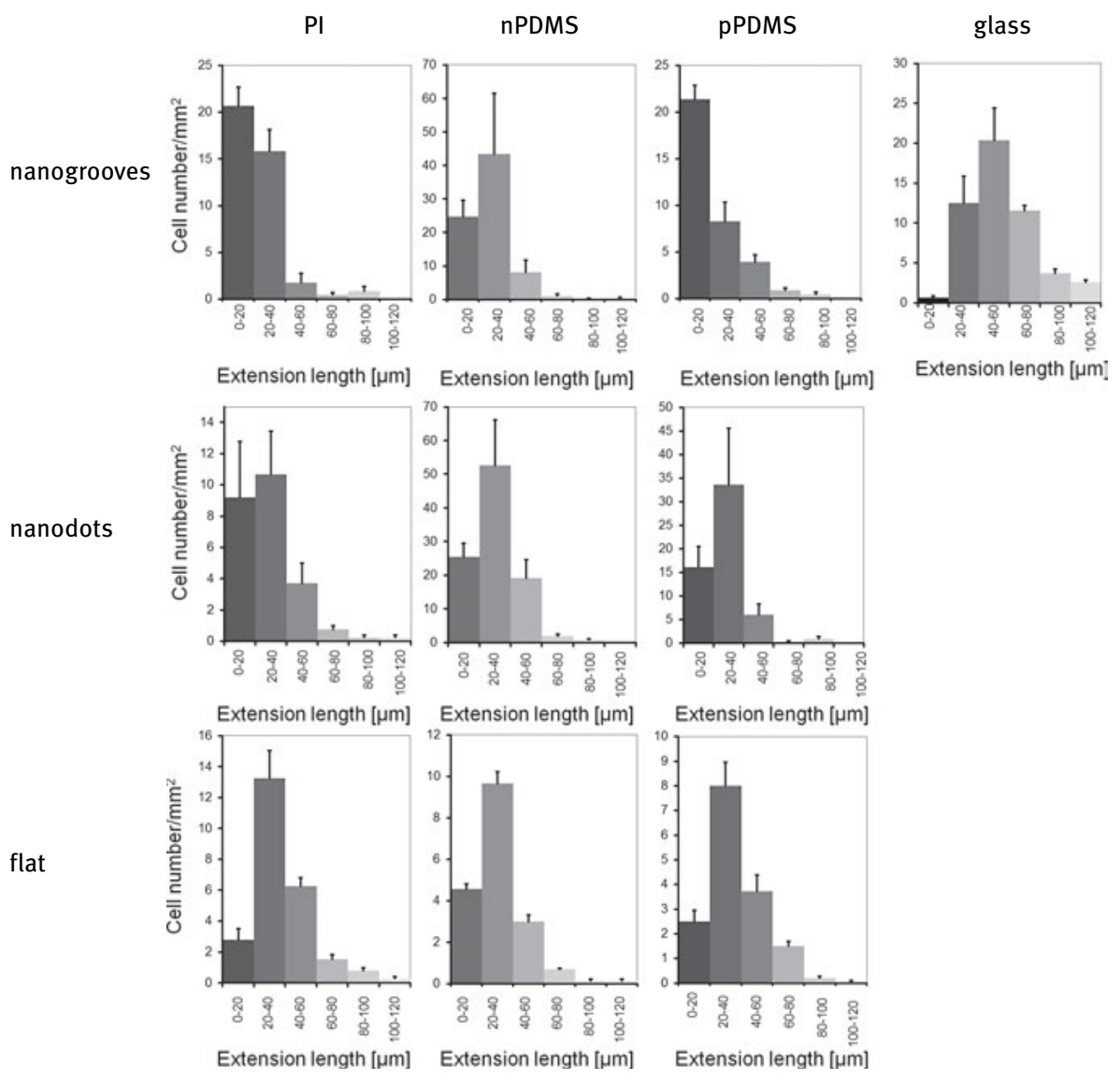

Fig. 2.8: Summary of cell numbers and length of cell extensions on nanogrooved and dotted polymer surfaces.

- increased cell numbers on nano patterned polymer surfaces compared to the unstructured polymer samples

- slightly decreased length of extensions on nanogrooved polymers (PI and positive PDMS cast) compared to unstructured polymer surfaces

- similar length distribution of extensions on nanodotted polymer surfaces compared to unstructured polymer samples, but increased length of extensions compared to grooved samples

- no clear effect of nanodotted lines on cell numbers and length of extensions.

These findings indicate that there is an influence of nanopatterns in the range of some hundred nanometers on cell numbers of neuronal cells as well as on orientation and length of cellular extensions. Further investigations are conducted with more sensitive cell types (MSCs). 


\subsubsection{Effect of nanotopography on MSC behavior}

MSCs isolated from the adipose tissue of healthy donors have been seeded on a polyimide foil patterned with grooves with a structure size of ca $300 \mathrm{~nm}$ obtained using multibeam interference (Fig. 2.9(a)). Polyimide is a biocompatible, inert plastic polymer which has been used for neuronal and sub-retinal implants. This material is also endowed with excellent mechanical properties such as low friction, high textile strength, resistance to wear and high flexibility [54-56]. No protein coating was used prior to the cell seeding in order to exclude the topographic influence of structures different to the nanogrooves and to improve the reproducibility of the experiments. Regarding cell adhesion there was no difference between the conventional tissue culture plastic, the non-structured PI used as control, and the nanogrooved PI; furthermore, this material also showed very low cytotoxicity since no relevant cell detachment or apoptosis was detected on any of the substrates. Interestingly, surface topography had a profound impact on cell shape: optical microscopy revealed that MSCs on the nanopatterned PI aligned parallel to the direction of the grooves and had an elongated morphology. Conversely, MSCs on the flat PI and on TCP displayed a random arrangement and no signs of elongation (Fig. 2.9 (b)). MSCs on the nanogrooved PI retained their alignment and elongated morphology even after having been successfully differentiated into adipocytes using an induction medium supplemented with specific chemicals and hormones (Fig. 2.9(c)). Scanning electronic microscopy images showed that cells developed nice filopodia and tightly interacted with the periodic nanogrooves (Fig. 2.9 (d)).

\subsection{Conclusion and outlook}

Multibeam interference represents a novel technology for surface nanostructuring applications. Compared to other techniques, MBI can directly ablate a substrate to generate smaller structures over a larger area at lower cost. Interestingly, nanostructures can have a deep impact on cellular biology and be integrated into biomedical devices. Neuronal cells have been reported to elongate along nanogrooves but not on nanodots or dotted nanogrooves. The results indicated that groove depth was more relevant than groove width in stimulating cell elongation. Furthermore, when interacting with primary MSCs, periodical nanogrooves supported their growth and allowed their differentiation towards adipocytes in addition to eliciting cell elongation. In conclusion, MBI represents a straightforward and flexible technique with a broad range of applications, particularly in life sciences, e.g. for the generation of smart biomaterials able to control complex cellular functions through specific surface patterns. 

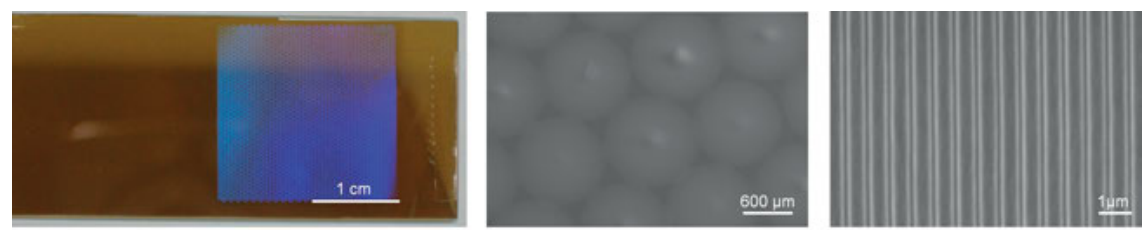

(a)
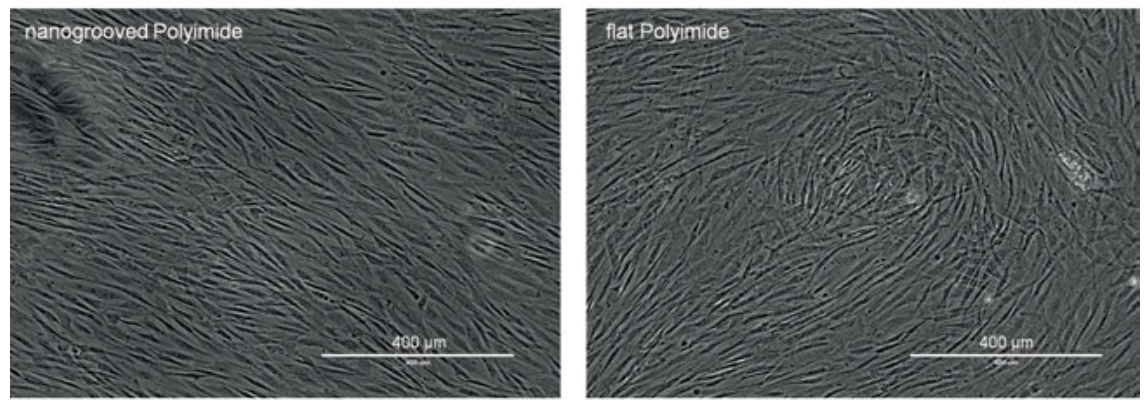

(b)
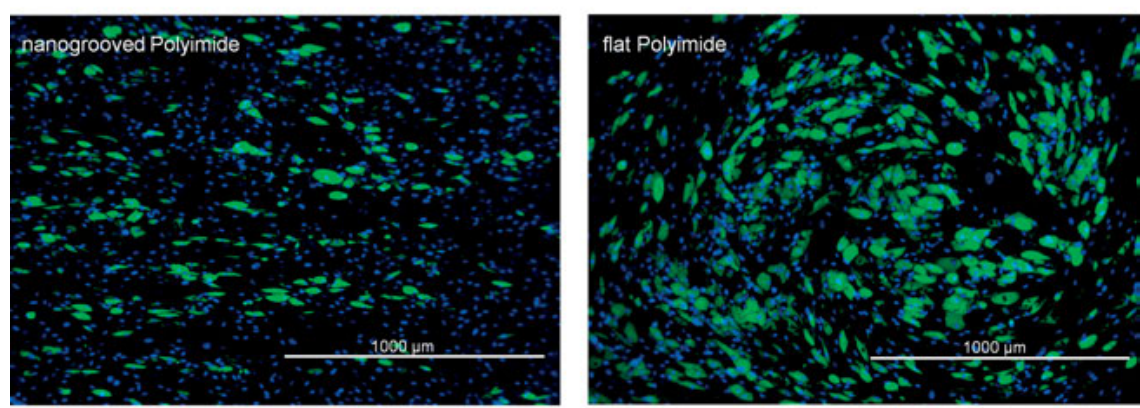

(c)
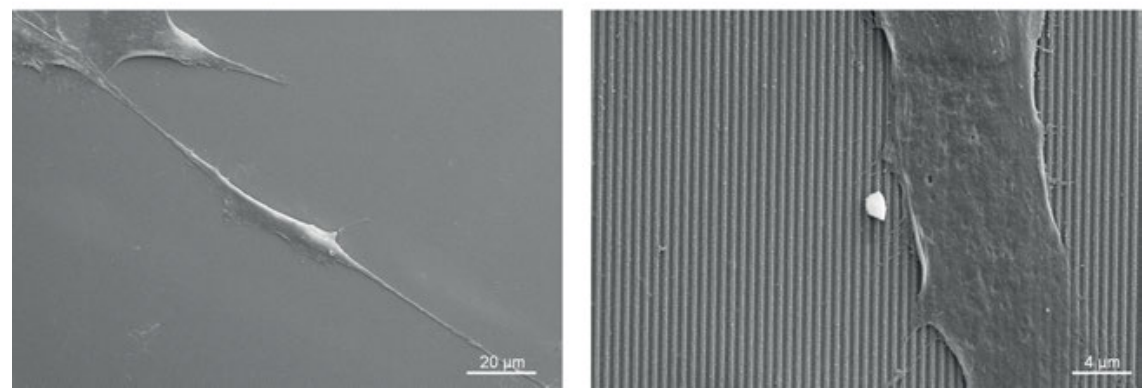

(d)

Fig. 2.9: (a) Photography of the nanogrooved polyimide foil with the structures depicted in blue due to the iridescence phenomenon (left), and SEM details of the nanogrooved areas (center and right). (b) Adipose tissue derived mesenchymal stem cells align parallel to the nanogrooves while displaying a random alignment on the non-structured surfaces. (c) Upon differentiation into adipocytes. MSCs retained their alignment. Fat droplets of the adipocytes are stained green with BODIPY while the nuclei are stained blue with DAPI. (d) Scanning electronic microscopy pictures of MSCs on the nanogrooved $\mathrm{PI}$. 


\section{References}

[1] Schellenberg A, Joussen S, Moser, K, Hampe, N, Hersch N, Hemeda H, Schnitker J, Denecke B, Lin, Q, Pallua N, Zenke M, Merke R, Hoffmann B, Wagner W. Matrix elasticity, replicative senescence and DNA methylation patterns of mesenchymal stem cells. Biomaterials, Issue 35, 2014, 6351-6358

[2] Laird DJ, von Andrian UH, Wagers AJ. Stem Cell Trafficking in Tissue Development, Growth, and Disease. Cell, Volume 132, Issue 4, 22 February 2008, 612-630

[3] Blau HM, Brazelton TR, Weimann JM. The Evolving Concept of a Stem Cell: Entity or Function? Cell, Volume 105, Issue 7, 29 June 2001, 829-841

[4] Weissman IL, Stem Cells: Units of Development, Units of Regeneration, and Units in Evolution. Cell, Volume 100, Issue 1, 7 January 2000, 157-168

[5] Morrison SJ, Shah NM, Anderson DJ. Regulatory Mechanisms in Stem Cell Biology. Cell, Volume 88, Issue 3, 7 February 1997, 287-298

[6] Knoblich JA. Mechanisms of Asymmetric Stem Cell Division. Cell, Volume 132, Issue 4, 22 February 2008, 583-597

[7] Jones L. Stem cells: So what's in a niche? Current Biology, Volume 11, Issue 12, 26 June 2001, R484-R486

[8] Morrison SJ, Spradling AC. Stem Cells and Niches: Mechanisms That Promote Stem Cell Maintenance throughout Life. Cell, Volume 132, Issue 4, 22 February 2008, 598-611

[9] Fuchs E, Tumbar T, Guasch G. Socializing with the Neighbors: Stem Cells and Their Niche. Cell, Volume 116, Issue 6, 19 March 2004, 769-778

[10] Caplan Al. Mesenchymal stem cells. Journal of Orthopaedic Research, Volume 9, Issue 5, 1991, 641-650

[11] da Silva ML, Chagastelles PC, Nardi NB. Mesenchymal stem cells reside in virtually all postnatal organs and tissues. Journal of Cell Science, Volume 119, Issue 11, June 2006, 2204-2213

[12] Phinney DG. Functional heterogeneity of mesenchymal stem cells: implications for cell therapy. Journal of Cell Biochem, Volume 113, Issue 9, September 2012, 2806-2812

[13] Ho AD, Wagner W, Franke W. Heterogeneity of mesenchymal stromal cell Preparations. Cytotherapy, Volume 10, Issue 4, 2008, 320-330

[14] Bianco P, Robey PG, Simmons PJ. Mesenchymal Stem Cells: Revisiting History, Concepts, and Assays. Cell Stem Cell, Volume 2, Issue 4, 10 April 2008, 313-319

[15] Dominici M, Le Blanc K, Mueller I, Slaper-Cortenbach I, Marini F, Krause D, Deans R, Keating A, Prockop DJ, Horwitz E. Minimal criteria for defining multipotent mesenchymal stromal cells. The International Society for Cellular Therapy position statement. Cytotherapy, Volume 8, Issue 4 August 2006, 315-317

[16] Nombela-Arrieta C, Ritz J, Silberstein LE. The elusive nature and function of mesenchymal stem cells. Nature Reviews Molecular Cell Biology, Volume 12, Issue 2, February 2011, 126-131

[17] Anguille S, Smits EL, Lion E, van Tendeloo VF, Berneman ZN. Clinical use of dendritic cells for cancer therapy. The Lancet Oncology, Volume 15, Issue 7, June 2014, e257-e267

[18] Pittenger MF, Mackay AM, Beck SC, Jaiswal RK, Douglas R, Mosca JD, Moorman MA, Simonetti DW, Craig S, Marshak DR. Multilineage Potential of Adult Human Mesenchymal Stem Cells. Science, Volume 284, Issue 5411, 2 April 1999, 143-147

[19] Uccelli A, Moretta L, Pistoia V. Mesenchymal stem cells in health and disease. Nature Reviews Immunology 8, September 2008, 726-736

[20] Macchiarini P, Jungebluth P, Go T, Asnaghi MA, Rees LE, Cogan TA, Dodson A, Martorell J, Bellini S, Parnigotto PP, Dickinson SC, Hollander AP, Mantero S, Conconi MT, Birchall MA. Clinical transplantation of a tissue-engineered airway. The Lancet, 13 December 2008, Vol. 372, Issue 9655, 2023-2030 
[21] Schellenberg A, Joussen S, Moser K, Hampe N, Hersch N, Hemeda H, Schnitker J, Denecke B, Lin Q, Pallua N, Zenke M, Merkel R, Hoffmann B, Wagner W. Matrix elasticity, replicative senescence and DNA methylation patterns of mesenchymal stem cells. Biomaterials, Volume 35, Issue 24, August 2014, 6351-6358

[22] Eyckmans J, Boudou T, Yu X, Chen CS. A Hitchhiker's Guide to Mechanobiology. Developmental Cell, Volume 21, Issue 1, 19 July 2011, 35-47

[23] Kim KM, Choi YJ, Hwang JH, Kim AR, Cho HJ, Hwang ES, Park JY, Lee SH, Hong JH- Shear stress induced by an interstitial level of slow flow increases the osteogenic differentiation of mesenchymal stem cells through TAZ activation. PLoS One, Volume 9, Issue 3, 21 March 2014, Article Number e92427

[24] Ballotta V, Smits AIPM, Driessen-Mol A, Bouten CVC, Baaijens FPT. Synergistic protein secretion by mesenchymal stromal cells seeded in 3D scaffolds and circulating leukocytes in physiological flow. Biomaterials, Volume 35, Issue 33, November 2014, 9100-9113

[25] De Lisio M, Jensen T, Sukiennik RA, Huntsman HD, Boppart MD. Substrate and strain alter the muscle-derived mesenchymal stem cell secretome to promote myogenesis. Stem Cell Research \& Therapy, 6 June 2014, Volume 5, Article 74

[26] Friedl G, Schmidt H, Rehak I, Kostner, G, Schauenstein K, Windhager R. Undifferentiated human mesenchymal stem cells (hMSCs) are highly sensitive to mechanical strain: transcriptionally controlled early osteo-chondrogenic response in vitro. Osteoarthritis and Cartilage, Volume 15, Issue 11, November 2007, 1293-1300

[27] Engler AJ, Sen S, Lee Sweeney H, Discher DE. Matrix Elasticity Directs Stem Cell Lineage Specification. Cell, Volume 126, Issue, 4, 25 August 2006, 677-689

[28] Lo CM, Wang HB, Dembo M, Wang YL. Cell movement is guided by the rigidity of the substrate. Biophysical Journey, Volume 79, Issue 1, July 2000, 144-152

[29] Conway A, Schaffer DV. Biophysical regulation of stem cell behavior within the niche. Stem Cell Research \& Therapy, Volume 3, 14 December 2012, Article 50

[30] Curtis A, Riehle M. Tissue engineering: the biophysical background. Physics in Medicine and Biology, Volume 46, Issue 4, April 2001, R47-R65.

[31] Humphries MJ. Integrin Structure. Biochemical Society Transactions, Volume 28, Part 4, August 2000, 311-340

[32] Giancotti FG, Ruoslahti E. Integrin Signaling. Science, Volume 285, Issue 5430, 13 August 1999, 1028-1033

[33] Tanentzapf G, Martin-Bermudo MD, Hicks MS, Brown NH. Multiple factors contribute to integrin-talin interactions in vivo. Journal of Cell Science, Volume 119, Issue 8, 15 April 2006, 1632-1644

[34] Geiger B, Spatz JP, Bershadsky AD. Environmental sensing through focal adhesions. Nature Reviews Molecular Cell Biology, Volume 10, Issue 1, January 2006, 21-33

[35] Scales TME, Parsons MP. Spatial and temporal regulation of integrin signalling during cell migration. Current Opinion in Cell Biology, Volume 23, Issue 5, October 2011, 562-568

[36] McBeath R, Pirone DM, Nelson CM, Bhadriraju K, Chen CS. Cell Shape, Cytoskeletal Tension, and RhoA Regulate Stem Cell Lineage Commitment. Developmental Cell, Volume 6, Issue 4, April 2004, 483-495

[37] Murphy WL, McDevitt TC, Engler AJ. Materials as stem cell regulators. Nature Materials, Volume 13, Issue 6, June 2014, 547-55

[38] Dalby MJ, Gadegaard N, Oreffo ROC. Harnessing nanotopography and integrin matrix interactions to influence stem cell fate. Nature Materials, Volume 13, Issue 6, June 2014, 558-569 
[39] McMurray RJ, Gadegaard N, Tsimbouri PM, Burgess KV, McNamara LE, Tare R, Murawski K, Kingham E, Oreffo ROC, Dalby MJ. Nanoscale surfaces for the long term maintenance of mesenchymal stem cell phenotype and multipotency. Nature Materials, Volume 10, Issue 8, 2010, 637-644

[40] Fiedler J, Özdemir B, Bartholomä J, Plettl A, Brenner RE, Ziemann P. The effect of substrate surface nanotopography on the behavior of multipotnent mesenchymal stromal cells and osteoblasts. Biomaterials, Volume 34, Issue 35, November 2013, 8851-8859

[41] Wang PY, Li WT, Yu J, Tsai WB. Modulation of osteogenic, adipogenic and myogenic differentiation of mesenchymal stem cells by submicron grooved topography. Journal of Materials Science: Materials in Medicine, Volume 23, Issue 12, December 2012, 3015-3028

[42] Wu YN, Law JBK, He AY, Low HY, Hui JHP, Lim CT, Zheng Yang Z, Lee EH. Substrate topography determines the fate of chondrogenesis from human mesenchymal stem cells resulting in specific cartilage phenotype formation. Nanomedicine: Nanotechnology, Biology and Medicine, Volume 10, Issue 7, October 2014, 1507-1516

[43] Nikkhah M, Edalat F, Manoucheri S, Khademhosseini A. Engineering microscale topographies to control the cell-substrate interface, Biomaterials, Volume 33, Issue 21, July 2012, 5230-5246

[44] Unadkat HV, Hulsman M, Cornelissen K, Papenburg BJ, Truckenmul"ler RK, Post GF, Uetz M, Reinders MJT, Stamatialis D, Van Blitterswijk CA, De Boer J. An algorithm-based topographical biomaterials library to instruct cell fate. Proceedings of the National Academy of Sciences of the United States of America, Volume 108, Issue 40, 2011, 16565-16570

[45] Ghaemi SR, Harding FJ, Delalat B, Gronthos S, Voelcker NH. Exploring the mesenchymal stem cell niche using high throughput screening. Biomaterials, Volume 34, Issue 31, October 2013, 7601-7615

[46] Robison RA. Moore's Law: Predictor and Driver of the Silicon Era. World Neurosurgery, Volume 78, Issue 5, November 2012, 399-403

[47] Burrow GM, Gaylord TK. Multi-Beam Interference Advances and Applications: NanoElectronics, Photonic Crystals, Metamaterials, Subwavelength Structures, Optical Trapping, and Biomedical Structures. Micromachines, Volume 2, Issue 2, June 2011, 221-257

[48] Beckemper S, Huang JT, Gillner A, Wang KY. Generation of Periodic Micro- and Nano-structures by Parameter-Controlled Three-beam Laser Interference Technique. JOURNAL OF LASER MICRO NANOENGINEERING, Volume 6, Issue 1, March 2011, 49-53

[49] Steger M, Hartmann C, Beckemper S, Holtkamp J, Gillner A. Fabrication of Hierarchical Structures by Direct Laser Writing and Multi-Beam-Interference. JOURNAL OF LASER MICRO NANOENGINEERING, Volume 8, Issue 3, December 2011, 210-215

[50] Hecht E. Optik. Oldenbourg, Munich, 2005, 624

[51] Steger M, Boes S, Thilker S, Gillner A. Measuring Method for the Interference Contrast of MultiBeam-Interference. JOURNAL OF LASER MICRO NANOENGINEERING, Volume 9, Issue 3. 2014

[52] Bremus-Koebberling E A, Beckemper S, Koch B, Seiler N. Laser Functionalization of Polymers to Create Bio-inspired Surfaces. International Bionic Engineering Conference 2011, 18-20 Sept. 2011, Boston, MA, USA.

[53] Bremus-Koebberling EA, Beckemper S, Koch B, Gillner A. Nano structures via laser interference patterning for guided cell growth of neuronal cells. Journal of Laser Applications 2012, 042013$1-6$.

[54] Klinge PM, Vafa MA, Brinker T, Brandis A, Walter GF, Stieglitz T, Samii M, Wewetzer K. Immunohistochemical characterization of axonal sprouting and reactive tissue changes after long-term implantation of a polyimide sieve electrode to the transected adult rat sciatic nerve. Biomaterials, Volume 22, Issue 17, September 2001, 2333-2343 
[55] Julien S, Peters T, Ziemssen F, Arango-Gonzalez B, Beck S, Thielecke H, Büth H, Van Vlierberghe S, Sirova M, Rossmann P, Rihova B, Schacht E, Dubruel P, Eberhart Zrenner E, Schraermeyer U. Implantation of ultrathin, biofunctionalized polyimide membranes into the subretinal space of rats. Biomaterials, Volume 32, Issue 16, June 2011, 3890-3898

[56] Rubehn B, Stieglitz T. In vitro evaluation of the long-term stability of polyimide as a material for neural implants. Biomaterials, Volume 31, Issue 13, May 2010, 3449-3458 
